Relations industrielles

Industrial Relations

\title{
Les relations patronales-syndicales au Québec, par Jean Boivin et Jacques Guilbault, Chicoutimi, Gaëtan Morin éditeur, 1982, 309 pp., ISBN 2-89105-087-8.
}

\section{Dimitri Weiss}

\section{Volume 37, numéro 4, 1982}

URI : https://id.erudit.org/iderudit/029316ar

DOI : https://doi.org/10.7202/029316ar

Aller au sommaire du numéro

Éditeur(s)

Département des relations industrielles de l'Université Laval

ISSN

0034-379X (imprimé)

1703-8138 (numérique)

Découvrir la revue

Citer ce compte rendu

Weiss, D. (1982). Compte rendu de [Les relations patronales-syndicales au Québec, par Jean Boivin et Jacques Guilbault, Chicoutimi, Gaëtan Morin éditeur, 1982, 309 pp., ISBN 2-89105-087-8.] Relations industrielles / Industrial Relations, 37(4), 969-970. https://doi.org/10.7202/029316ar

Tous droits réservés @ Département des relations industrielles de l'Université Laval, 1982
Ce document est protégé par la loi sur le droit d'auteur. L'utilisation des services d’Érudit (y compris la reproduction) est assujettie à sa politique d'utilisation que vous pouvez consulter en ligne.

https://apropos.erudit.org/fr/usagers/politique-dutilisation/ 
the Webbs, Commons, Perlman, Dunlop, Kerr et al., etc., is devoid of much of the essence of institutionalism.

The body of this book is devoted to process. Chapter 6 provides an excellent discussion of union organizing; the activities of the parties and the Board throughout organizing campaigns, unit determinations and (de)certifications are explained in a way that anticipates student curiousity and puts the informatio into a comprehensible framework. An illuminating discussion of union democracy is also included. Chapters 7-10 deal with bargaining theory, structures and issues. An extensive section on bargaining structure is provided. The author utilizes Walton and McKersie's seminal work to good effect in explaining bargaining subprocesses. A book with such a sharp focus upon process would have benefited from a stronger emphasis upon bargaining strategies and tactics to augment the accompanying simulation case. Chapter 12 introduces the concept of unionmanagement cooperation and outlines approaches which have been made to implement it within and beyond the confines of collective bargaining. The chapter could have been improved through greater use of comparative material.

The final portion of the book is given over to chapters on public sector and healthcare labour relations, equal employment opportunities, and challenges facing collective bargaining, the bulk of which has little applicability to Canada.

Overall, the book is well organized and very readable, making effective use of flow charts to clarify sequencing and contingencies. The work is not encyclopedic; it is, in fact, sketchy in a number of areas. It does present current behavioral research and timely topics. One might have expected that the basic flaw, absence of a strong structure section, to have been remedied in this revision. Since it has not been, the book suffers.

Kurt WETZEL University of Saskatchewan

\section{Les relations patronales-syndicales au Qué-} bec, par Jean Boivin et Jacques Guilbault, Chicoutimi, Gaëtan Morin éditeur, 1982, 309 pp., ISBN 2-89105-087-8.

Il faut être redevable aux deux auteurs d'avoir mis à la disposition des formateurs et des "preneurs de formation», qu'il s'agisse d'étudiants «classiques» ou d'autres, un ouvrage d'initiation dont la nécessité et l'utilité auront déjà été, vraisemblablement, démontrées à l'heure de la parution de la présente note.

Jean Boivin et Jacques Guilbault, en qualifiant d'emblée leur livre comme «un exercice de vulgarisation» visant, par une information le plus simplement exprimée et en même temps substantielle, un large public, expliquent par ce souci le choix du titre du volume, de préférence à celui de relations du travail. Du même coup, étant donné les connotations de l'expression "relations patronales-syndicales», ils mettent leur manuel en perspective historique, vertu cardinale pour introduire à un domaine qui - peut-être plus dans mon pays que dans le leur - engendre, même parmi certains acteurs du système, des malentendus superflus dus au manque de notions d'histoire du mouvement syndical, unies à celle, parallèle du business.

Mais, en faisant leur affaire de la description de l'interaction des partenaires sociaux au Québec, ils ont, dès l'abord, le souci de montrer qu'ils ne prétendent pas couvrir par les seuls rapports collectifs le domaine, plus vaste des relations industrielles. Et il n'est pas étonnant de voir deux professeurs du département qui a, le premier au Québec, me semble-t-il, donné une consistance institutionnelle à l'enseignement de cette multidiscipline (visant à l'interdisciplinarité), rappeler l'importance, non moindre, des sciences du comportement et de la gestion des ressources humaines dans sa structuration et son développement.

Boivin et Guilbault ont raison de dire que tout ce qui est «travail» n'est pas forcément rapportable aux relations industrielles. Certes, la pluridisciplinarité ne signifie guère 
que tout ce qui tient, par exemple, à l'économie, au droit, à la sociologie ou à la psychologie du travail soit nécessairement afférent au champ des relations industrielles. Ce qui a comme première conséquence une dispersion de la recherche conduisant à affronter parfois marginalement des thèmes typiques et porteurs.

La première vérité pour les deux compères est que la nature des relations patronalessyndicales est, par excellence, conflictuelle. Et ils n'oublient pas de confronter ici la manifestation la plus "visible» et spectaculaire du conflit ouvert qu'est la grève aux différentes formes de conflit latent, tel l'absentéisme. Dans ce dernier domaine, l'inspirateur est A.E.D. Hare. Dans le premier, mon collègue Jean Savatier, juriste de l'Université de Poitiers (qu'une coquille obstinée a transformé en... coordonnier).

Conflit collectif-négociation collective étant le concept-couple central des relations du travail, les auteurs nous présentent, dans le passage de la première notion à l'autre, les acteurs: organisations syndicales et patronales - histoire, structure et idéologie - , qui se lit avec le plus vif intérêt, en réservant à l'entière dernière partie la négociation collective.

La lecture de celle-ci ne pourrait, pour un Français, ne pas donner lieu, surtout en ce moment, à un rapprochement avec le débat hexagonal, très vif, autour du concept de négociation collective et de l'obligation de négocier, sur le point d'être introduite dans l'arsenal législatif (dix-huit ans après le Québec).

Nombre de propos des auteurs auront pu être repris par le ministre français du Travail dans l'oeuvre de persuasion qu'il mène depuis l'année dernière pour imposer un mode contractuel de confrontation. Et lorsque Boivin et Guilbault présentent, à la suite de l'expérience accumulée par nombre de négociateurs, syndicalistes et employeurs, certaines lignes de conduite qui font, notamment, apparaître la négociation comme un processus pédagogique, voire maïeutique, ce n'est pas autre chose que veulent instaurer, de ce côtéci de l'Atlantique, les promoteurs du projet de loi sur la négociation collective, actuellement en discussion au Parlement. Ou encore lorsqu'ils disent qu'«un employeur doit accepter la réalité syndicale sans réserve et la considérer comme une force constructive».

"Il faudra que les entreprises acceptent de vivre désormais à l'heure contractuelle», écrivait récemment Michel Rocard. "L'indépendance des parties n'est pas en cause. Mais il n'y aura de société négociée que si les partenaires sociaux savent se reconnaître dans leur légitimité réciproque. Les entrepreneurs doivent comprendre que la solidarité dans l'entreprise ne peut se décréter, mais doit naître de la reconnaissance réciproque. Les syndicats doivent comprendre que la création de nouveaux emplois exige des entrepreneurs performants. La société française doit assumer le fait qu'il n'y aura de mobilisation industrielle et d'effort collectif que derrière des organisations syndicales fortes et respectées.»

Ceci vaut, toutes proportions gardées, tout aussi bien pour la France que pour le Québec. Boivin et Guilbault y auront, sans doute, entièrement souscrit. Ce qui prouverait que les distances ne sont pas aussi énormes qu'on s'applique, depuis trop longtemps, à le dire. Et qu'entre le Québec nord-américain et la France européenne une étude comparée - d'abord, peut-être, sur les conventions collectives - reste à faire.

J'ai découvert parmi les références, pas très nombreuses, du livre, le nom d'un ancien étudiant de Jean Boivin - qui fut aussi le mien, pour un court laps de temps - , P.-P. Bilodeau. J'espère que d'autres, parmi ses élèves actuels, seront tentés par un regard comparé. En attendant, «Les relations patronales-syndicales au Québec» leur ouvriront la voie, excellemment, à des tentations futures.

Dimitri WEISS

Université du Maine et Institut d'Administration des Entreprises, Paris 